\section{Fungal keratitis in the Republic of Ireland}

\begin{abstract}
Purpose Fungal keratitis is relatively rare in temperate climates with only one previous population-based study, which reported a minimum annual incidence of 0.32 per million population in the United Kingdom. We performed a nationwide study in the Republic of Ireland of cases over a 6-year period to establish the incidence, risk factors, treatment, and outcomes of fungal keratitis. Patients and methods All corneal scraping specimens positive for fungal species over a 6-year period were identified at the microbiology departments of the seven main ophthalmology units in the Republic of Ireland. Census data was used to establish national minimum annual incidence.

Results Forty-two eyes were treated for confirmed fungal keratitis during the 6-year period. This resulted in a minimum incidence of $\mathbf{1 . 5 3}$ cases per million population per year. Twenty-eight of 42 cases $(67 \%)$ were due to filamentary fungi, with the most common species being Aspergillus. Pre-existing ocular surface disease was the most common risk factor constituting $42.9 \%$; $26.2 \%$ underwent keratoplasty. No eyes underwent evisceration. Two eyes resulted in a level of vision of no perception of light. The mean LogMAR acuity in the remainder of cases was 0.89 with a range from -0.10 to 4.00 .

Conclusion This study demonstrates a much higher minimum annual incidence than previously reported. The causative organism is highly related to risk factor. The range of visual outcomes remains broad in all subgroups. The wide range of outcomes and treatments used highlights the need for better evidence-based guidelines for fungal keratitis.
\end{abstract}

Eye (2017) 31, 1427-1434; doi:10.1038/eye.2017.82; published online 19 May 2017

\section{Introduction}

In the tropics, $21-62 \%$ of all culture positive microbial keratitis cases are caused by fungi, predominantly by filamentary fungi of the
S Farrell ${ }^{1}$, E McElnea ${ }^{2}$, S Moran ${ }^{3}$, S Knowles ${ }^{4}$ and CC Murphy ${ }^{1}$

Fusarium and Aspergillus species. It most commonly occurs in men and the main risk factor is trauma. ${ }^{1-13}$ Many large case series from a variety of tropical countries have been reported, in contrast to a small number of singleinstitution case series from temperate areas of the world including the United States, ${ }^{14-17}$ Australia, ${ }^{18}$ and the United Kingdom. ${ }^{19,20}$ In temperate climates, it appears to be a rare disease with only $1-5 \%$ of cases of microbial keratitis due to fungi. ${ }^{14,18,19}$ Though less common, it remains a severe disease requiring therapeutic keratoplasty in $12-48 \%$ of cases and enucleation in up to $6 \%$ of cases. $14,16,18,19$ Chronic ocular surface disease and contact lens wear $^{12}$ are the main risk factors in temperate areas. Affected individuals tend to be older, and a higher proportion are female. ${ }^{15,21}$ Until recently, Candida was recognized as the most commonly identified species in temperate areas, ${ }^{14,15}$ however a recent study from London has reported that the microbiological patterns of fungal keratitis are changing. In that study $70 \%$ of cases of fungal keratitis were due to filamentary infections, with Fusarium species the most common species. ${ }^{20}$

Only one population-based study of the prevalence, risk factors, causative organisms, and clinical outcomes of fungal keratitis in temperate regions has been reported to-date. ${ }^{22}$ The aim of this study is to report the epidemiological, clinical, and microbiological features of all confirmed fungal keratitis cases in the Republic of Ireland, a temperate country, in a 6-year population-based study.

\section{Materials and methods}

\section{Inclusion criteria}

All corneal scraping specimens positive for fungal species by culture, microscopy, or molecular biological analysis over a 6-year period were identified at the microbiology departments of the seven major ophthalmology units in the Republic of Ireland. All such patients were included in this study.
${ }^{1}$ Department of Ophthalmology, Royal Victoria Eye and Ear Hospital, Dublin, Ireland

${ }^{2}$ Department of Ophthalmology, Mater Misericordiae University Hospital, Dublin, Ireland

${ }^{3}$ Department of Ophthalmology, Cork University Hospital, Cork, Ireland

${ }^{4}$ Department of Microbiology, Royal Victoria Eye and Ear Hospital, Dublin, Ireland

Correspondence: S Farrell, Department of Ophthalmology, Royal Victoria Eye and Ear Hospital, Adelaide Road, Dublin D02XK51, Ireland Tel: +353 1863870412 ; Fax: +35316761858. E-mail: stephenfarrell@ fastmail.com

Received: 4 October 2016 Accepted in revised form: 24 March 2017 Published online: 19 May 2017 


\section{Exclusion criteria}

Patients were excluded from further analysis if the treating clinician decided that the clinical picture was not consistent with fungal keratitis and the infection resolved without any antifungal treatment. In such cases, the positive fungal result was deemed to be non-pathogenic or due to a contaminant.

\section{Clinical and microbiological record review}

Demographic details recorded included patient age and gender. Risk factors for fungal infection including history of recent trauma to the eye, contact lens use, previous corneal surgery, pre-existing ocular surface disease, use of topical steroids at the time of onset of symptoms, use of other topical ophthalmic medications, and recent travel to the tropics were recorded. Results of microscopy, fungal culture or fungal RNA, or DNA assays were documented. Microscopy was performed using Gram stain and fungal culture was performed using Sabouraud dextrose agar as standard in all units. Culture with blood agar and chocolate agar plates was also routinely performed. In vivo confocal microscopy was not available in any unit. The species of fungus cultured and the reports of sensitivities to antifungal agents were recorded. All topical, intrastromal, intracameral, oral, or intravenous antifungal agents used and all surgical treatments employed to control the infection were recorded. Date of onset of symptoms based on history and date of commencement, and discontinuation of antifungal treatment was documented. Visual outcome was recorded as the best recorded visual acuity either unaided, with refractive correction or using pinhole following resolution of the infection and discontinuation of antifungal medication. All visual acuities were converted to LogMAR and patients with no perception of light or perception of light only were not designated a

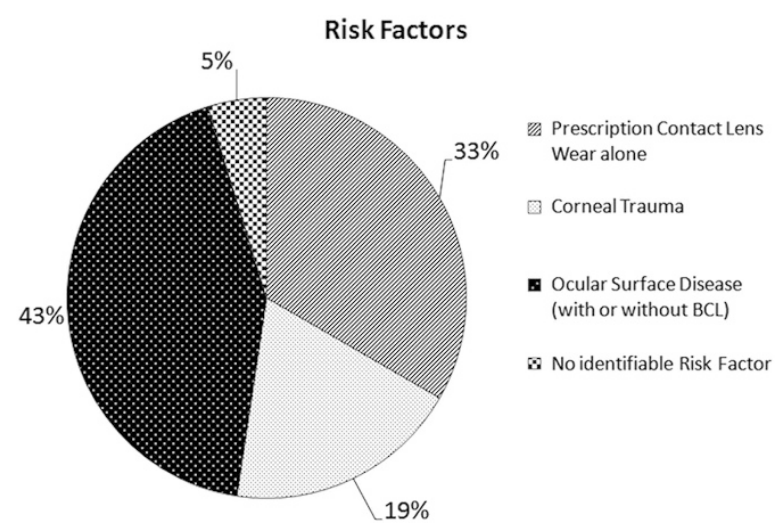

Figure 1 Risk factors for the development of fungal keratitis.
LogMAR visual acuity, but were reported separately in the results as per Holladay. ${ }^{23}$

Multivariable regression analysis to investigate associations between age, history of chronic ocular surface disease, history of contact lens wear and history of trauma, and risk of developing fungal keratitis due to yeast $v$ s filamentary species was performed and a $P$-value of $<0.05$ was considered statistically significant.

\section{Epidemiological analysis}

The incidence of confirmed fungal keratitis was calculated using the National Census for the Republic of Ireland in 2011, which recorded the population at 4588252 . The minimum incidence of fungal keratitis is reported as the number of cases per year per million population. ${ }^{24}$

\section{Results}

\section{Patient demographics and incidence of fungal keratitis}

Forty-two eyes of 42 patients were treated for confirmed fungal keratitis during the 6-year period. From this we calculated a minimum incidence of 1.53 cases per million population per year. Twenty-four (57.1\%) patients were male, 18 (42.9\%) were female. The age at time of diagnosis ranged from 19 to 85 years with a mean age of 47.4 years (SD 20.7).

\section{Risk factors for fungal keratitis}

The distribution of risk factors is illustrated in Figure 1. In 2 patients $(4.8 \%)$, no risk factor for fungal keratitis was identified.

Ocular surface disease. Eighteen eyes (42.9\%) suffered from pre-existing ocular surface disease. These included 8 eyes $(19.0 \%)$, which had previously undergone penetrating keratoplasty. The indications for keratoplasty included scarring following trauma, Stevens-Johnsons syndrome, Hurlers syndrome, rheumatoid melt, endothelial dystrophy, interstitial keratitis, herpes simplex keratitis, and lattice dystrophy (one for each indication). The other ocular surface conditions included corneal exposure, previous bacterial keratitis, previous acanthamoeba keratitis, recent corneal collagen crosslinking, ectropion, pseudophakic bullous keratopathy (one for each of these) and the use of preserved topical anti-glaucoma medications (two eyes).

Topical corticosteroid use. Twelve patients (28.6\%), including all eight of the patients with a history of corneal transplantation and four other patients with other ocular 
Table 1 Organisms cultured

\begin{tabular}{|c|c|c|c|c|}
\hline Organism & Entire cohort & Ocular surface disease & Contact lens & Trauma \\
\hline Aspergillus & $14(33.3 \%)$ & $5(27.8 \%)$ & $2(14.3 \%)$ & $5(62.5 \%)$ \\
\hline Candida & $11(26.2 \%)$ & $9(50.0 \%)$ & $1(7.1 \%)$ & $1(12.5)$ \\
\hline Fusarium & $8(19.0 \%)$ & $0(0.0 \%)$ & $8(57.1 \%)$ & $0(0.0 \%)$ \\
\hline Aspergillus and Candida & $1(2.4 \%)$ & $1(5.6 \%)$ & $0(0.0 \%)$ & $0(0.0 \%)$ \\
\hline Aspergillus and Fusarium & $1(2.4 \%)$ & $0(0.0 \%)$ & $1(7.1 \%)$ & $0(0.0 \%)$ \\
\hline Candida and Fusarium & $1(2.4 \%)$ & $0(0.0 \%)$ & $1(7.1 \%)$ & $0(0.0 \%)$ \\
\hline Phoma & $1(2.4 \%)$ & $0(0.0 \%)$ & $1(7.1 \%)$ & $0(0.0 \%)$ \\
\hline Basidomycetes & $1(2.4 \%)$ & $1(5.6 \%)$ & $0(0.0 \%)$ & $0(0.0 \%)$ \\
\hline Mucor & $1(2.4 \%)$ & $0(0.0 \%)$ & $0(0.0 \%)$ & $1(12.5 \%)$ \\
\hline Unspecified & $3(7.1 \%)$ & $2(11.1 \%)$ & $0(0.0 \%)$ & $1(12.5 \%)$ \\
\hline
\end{tabular}

Aspergillus was the most common species cultured in the entire group. The most common organism cultured varied based on the underlying risk factor.

surface disorders, were taking topical steroids at the time they developed fungal keratitis.

Contact lens wear. A total of 17 patients (40.5\%) were wearing contact lenses at the time fungal keratitis developed. Three patients $(7.1 \%)$ were wearing a bandage contact lens for ocular surface disease. Fourteen patients $(33.3 \%)$ wore prescription contact lenses with no other risk factor. The contact lenses were daily disposable in five cases, monthly disposable in seven cases, extended wear in one case, and in one case the contact lens type was not specified. Two patients had worn their daily contact lenses overnight and one patient had recently been swimming with contact lenses in situ.

Trauma. Eight eyes (19.0\%) developed fungal keratitis following corneal trauma. The cause of the trauma included a fingernail (three eyes), wood (two eyes), a tree branch (one eye), silage (processed grass) (one eye), and a metallic foreign body (one eye).

The mean age of the patient at time of diagnosis was 62 (SD 21.2) years in the ocular surface disease group, 40 (15.8) years in the contact lens group, and 51 (18.6) years in the trauma group. The mean time from the onset of symptoms to commencement of treatment was 18 days (range 0-98 days, SD 25.9 days) in the ocular surface disease group, $20(4-55,19.0)$ days in the contact lens group, and $26(0-104,36.4)$ days in the trauma group.

Multivariable regression analysis to investigate associations between age, history of chronic ocular surface disease, history of contact lens wear and history of trauma, and risk of developing fungal keratitis due to yeast $v$ s filamentary species resulted in $P$-values of 0.67 , $0.90,0.83,0.51$, respectively, showing that the trends described above do not reach statistical significance in this series of 42 cases.

\section{Microbiology results}

Thirty-eight patients $(90.5 \%)$ had positive fungal culture. Two cases $(4.8 \%)$ had positive microscopy with negative culture. These cases had signs consistent with fungal keratitis. One case $(2.5 \%)$ had positive microscopy and culture, and 1 case $(2.5 \%)$ had positive fungal PCR assay with negative microscopy and culture. Microscopy was performed using Gram stain. $\mathrm{KOH}$ staining was not performed in any of the units.

The organisms identified are shown in Table 1. Overall, Candida species were identified in 13 cases (30.9\%), filamentary fungi in 28 cases $(66.7 \%)$, and in 3 cases $(7.1 \%)$ the fungal species was unspecified. In 2 cases (4.8\%) a bacterial organism was grown in addition to a fungal species. In one case a propionibacterium species was cultured in addition to Aspergillus in a patient with chronic ocular surface disease. In another case a corynebacterium species was cultured in addition to Aspergillus in a patient with a history of ocular trauma.

The causative fungus varied depending on the underlying risk factor, with Candida species most common in the ocular surface disease group, Fusarium species most common in the contact lens group, and Aspergillus species most common in the trauma group. Further details are described in Table 1.

Sensitivity testing was reported in 16 of the cases, as shown in Table 2. All species showed at least intermediate sensitivity to voriconazole. One Fusarium species was resistant to amphotericin.

\section{Antifungal treatment}

All patients in this study received topical antifungal therapy, as summarized in Table 3. The most common topical treatment was a combination of voriconazole and amphotericin with 14 patients (33.3\%) receiving this 
Table 2 Results of sensitivity testing performed

\begin{tabular}{|c|c|c|c|}
\hline Organism species & Sensitive & Intermediate & Resistant \\
\hline Aspergillus & $\begin{array}{l}\text { Voriconazole } \\
\text { Amphotericin }\end{array}$ & & \\
\hline Aspergillus & Voriconazole & & \\
\hline Aspergillus & Voriconazole & & \\
\hline Aspergillus & Amphotericin & & \\
\hline Aspergillus & & $\begin{array}{l}\text { Econazole } \\
\text { Miconazole }\end{array}$ & \\
\hline Candida & $\begin{array}{l}\text { Amphotericin } \\
\text { Flucytosine } \\
\text { Voriconazole } \\
\text { Fluconazole }\end{array}$ & & \\
\hline Candida & $\begin{array}{l}\text { Amphotericin } \\
\text { Fluconazole }\end{array}$ & & \\
\hline Candida & $\begin{array}{l}\text { Fluconazole } \\
\text { Voriconazole } \\
\text { Caspofungin }\end{array}$ & & \\
\hline Candida & $\begin{array}{l}\text { Amphotericin } \\
\text { Voriconazole } \\
\text { Fluconazole }\end{array}$ & & \\
\hline Candida & $\begin{array}{l}\text { Fluconazole } \\
\text { Amphotericin } \\
\text { Voriconazole } \\
\text { Flucytosine }\end{array}$ & & \\
\hline Candida & $\begin{array}{l}\text { Fluconazole } \\
\text { Amphotericin }\end{array}$ & & \\
\hline Candida & $\begin{array}{l}\text { Fluconazole } \\
\text { Voriconazole } \\
\text { Itraconazole } \\
\text { Flucytosine }\end{array}$ & & \\
\hline Fusarium & $\begin{array}{l}\text { Posaconazole } \\
\text { Voriconazole } \\
\text { Ketoconazole } \\
\text { Amphotericin }\end{array}$ & & $\begin{array}{l}\text { Fluconazole } \\
\text { Caspofungin }\end{array}$ \\
\hline Fusarium & Voriconazole & & \\
\hline Fusarium & & $\begin{array}{l}\text { Econazole } \\
\text { Voriconazole }\end{array}$ & $\begin{array}{l}\text { Amphotericin } \\
\text { Itraconazole } \\
\text { Posaconazole }\end{array}$ \\
\hline Fusarium & Amphotericin & Voriconazole & \\
\hline
\end{tabular}

regimen. Seven patients $(17 \%)$ were treated with amphotericin drops alone, 7 patients $(16.7 \%)$ with voriconazole drops alone, and 5 patients $(11.9 \%)$ with natamycin drops alone. The remaining nine eyes were treated with a regimen including topical econazole, miconazole, and natamycin.

In addition to topical therapy, 6 eyes (14.3\%) received anterior segment locally injected voriconazole. Four of these received intrastromal and intracameral voriconazole, one patient received intracameral voriconazole alone, and 1 patient received intrastromal voriconazole alone. Four of these six eyes subsequently underwent penetrating keratoplasty. Two eyes (4.8\%) received intravitreal amphotericin injections. The intravitreal injections were given in conjunction with systemic antifungal agents in two cases of fungal keratitis,
Table 3 Summary of topical antifungal regimens used

\begin{tabular}{lc}
\hline Topical antifungal regimen & No. of eyes (\%) \\
\hline Voriconazole and amphotericin & $14(33.3 \%)$ \\
Amphotericin & $7(16.7 \%)$ \\
Voriconazole & $7(16.7 \%)$ \\
Natamycin & $5(11.9 \%)$ \\
Econazole & $1(2.4 \%)$ \\
Miconazole & $2(4.8 \%)$ \\
Econazole and amphotericin & $1(2.4 \%)$ \\
Econazole and voriconazole & $1(2.4 \%)$ \\
Amphotericin, econazole, and voriconazole & $1(2.4 \%)$ \\
Miconazole and amphotericin & $1(2.4 \%)$ \\
Natamycin and amphotericin & $1(2.4 \%)$ \\
Miconazole and voriconazole & $1(2.4 \%)$ \\
\hline
\end{tabular}

which presented with hypopyon due to Aspergillus species.

Twenty-two patients received systemic antifungal agents $(52.4 \%)$. Two patients received intravenous voriconazole and one patient was treated with intravenous amphotericin. Ten patients received oral voriconazole. Three patients received oral fluconazole. Three patients received both oral voriconazole and amphotericin B. One patient received voriconazole, fluconazole, and miconazole.

\section{Surgical treatment}

Eleven eyes (26.2\%) underwent therapeutic corneal transplantation, which included 10 penetrating keratoplasties and 1 deep anterior lamellar keratoplasty. One of these patients subsequently had a Boston Keratoprosthesis inserted. No eyes underwent enucleation or evisceration. Two eyes underwent tarsorrhaphy and three eyes underwent amniotic membrane transplantation. Of the eyes of patients who received systemic treatment, 8 of $22(36.4 \%)$ underwent corneal graft. Of the eyes of patients who did not receive systemic treatment, 3 of $20(15.0 \%)$ underwent corneal graft.

Nine of the 11 patients (82\%) who underwent keratoplasty received topical antifungal agents postoperatively. The mean duration of topical treatment in these patients was 111 days (range 39-340 days, SD 100 days). One patient who received topical antifungal treatment post-operatively was found to have residual fungal infection in the remaining host cornea day 1 postoperatively. That patient received oral antifungal treatment for a further 36 days and topical antifungal agents until they underwent a second penetrating keratoplasty 4 months after the initial graft. Topical antifungal agents were continued for 210 days after the second graft with no recurrence of fungal infection. Apart 
a

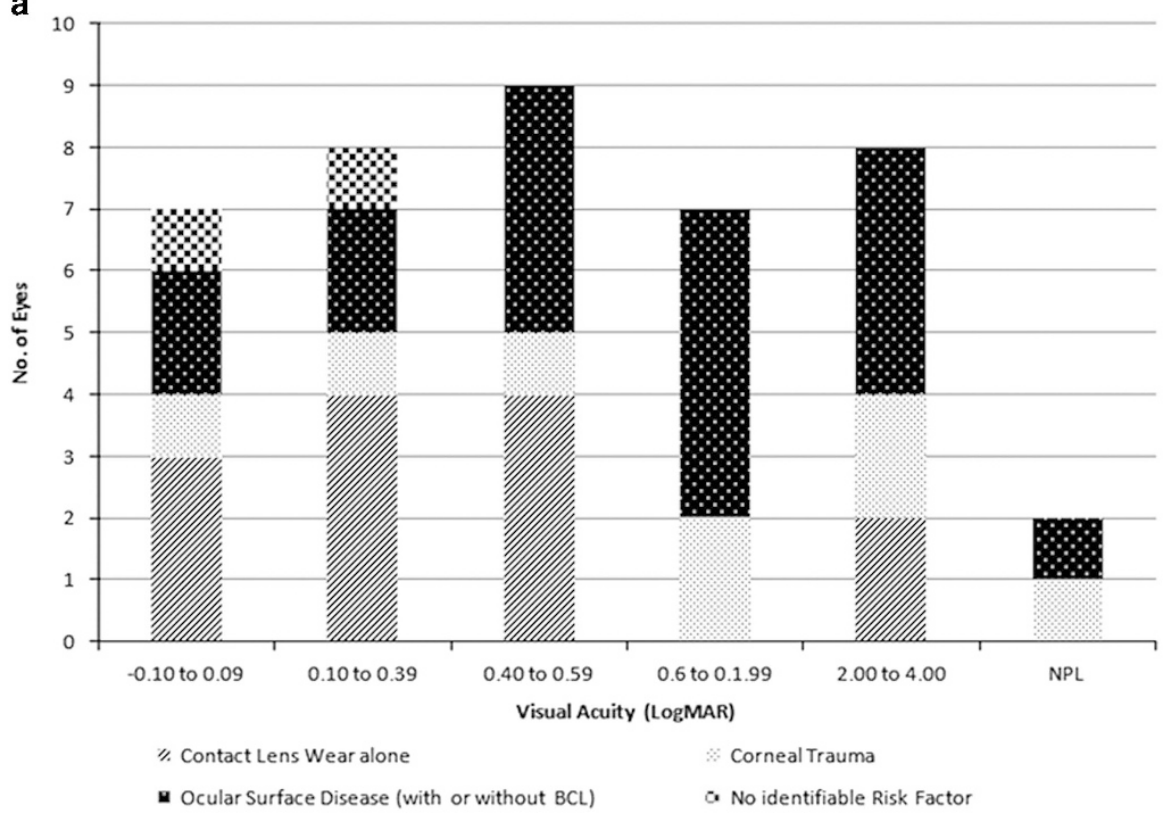

b

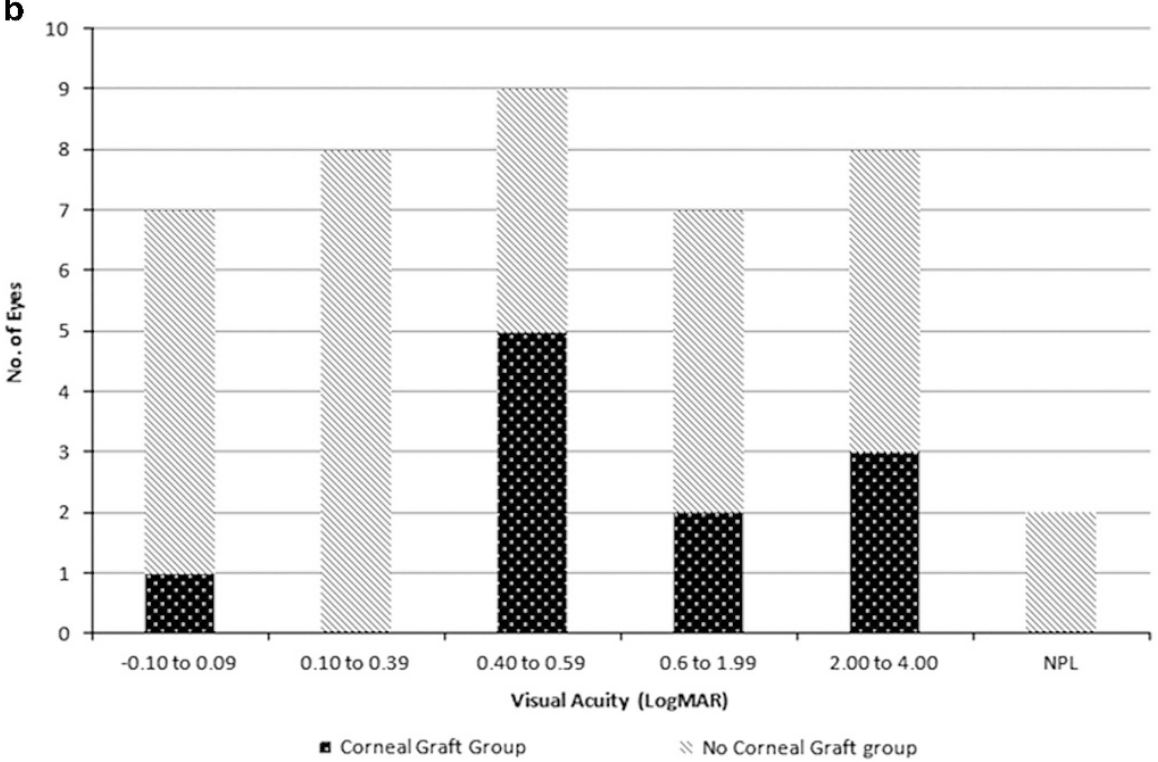

Figure 2 (a) Final best visual acuity. Frequency of best LogMAR visual acuity is displayed. Each subgroup based on risk factor for fungal keratitis is displayed with a different pattern of shading. (b) Final best visual acuity. Frequency of best LogMAR visual acuity is displayed. Patients are divided based on whether they underwent corneal graft or not.

from that patient, only one patient who underwent keratoplasty received oral antifungal treatment postoperatively and that was for a duration of 7 days with no recurrence of fungal infection. No other patients who were treated with topical antifungal agents postoperatively had a recurrence of fungal infection. One of the two patients who did not receive post-operative antifungal treatment had recurrent fungal infection in the graft 2 months post-operatively. That patient underwent repeated penetrating keratoplasty 5 months after the initial graft and was treated with topical antifungal agents for 3 weeks with no recurrence in the second graft.

\section{Visual outcomes}

The best recorded visual acuity following resolution of the infection and completion of antifungal treatment was recorded. The mean (range, SD) LogMAR visual acuity 
overall was 0.89 (-0.10 to $4.00,1.19)$. The mean (range, $\mathrm{SD})$ visual acuity for isolated infections with Aspergillus, Fusarium, and Candida species was 0.73 (-0.10 to 6.0, 1.18), $0.19(-0.10$ to $0.5,0.24)$, and $1.23(0.0-4.0,0.24)$. The mean (range, SD) visual acuity for the three mixed fungal infections, the three unspecified infections, and the three other filamentary fungi were $2.47(0.40-4.00(1.86)), 0.77$ (0.00-2.00 (1.08)), and $0.53(0.40-0.80,0.23)$. Two eyes resulted in no perception of light vision; both of these were caused by Aspergillus species; one following trauma to the eye; the other associated with pre-existing ocular surface disease. Both patients with no perception of light vision had received systemic antifungal agents. Of the remaining eyes, visual acuity of eyes of patients who received systemic treatment was $0.94(0.00-4.00$ (1.13)) and of patients who did not receive systemic treatment was 0.84 (-0.10 to $4.00(1.28))$. Visual acuity of patients who did receive corneal grafts was 1.14 (0.00-4.00 (1.29)). The two eyes with no perception of light vision had not undergone corneal transplant. Of the remaining eyes, visual acuity of eyes of patients who did not receive corneal grafts was 0.79 (-0.10 to $4.00(1.15))$.

Figure 2a shows the final best recorded visual acuity in relation to risk factor for fungal keratitis. It demonstrates that, while the two patients with no identifiable risk factor had a good visual result, all other risk factor groups had a very broad range of outcomes ranging from better than 0.1 to worse than 2.0. Similarly both the graft and nongraft group had a very broad range of outcomes as demonstrated in Figure 2b.

\section{Discussion}

This study estimates the minimum incidence of Fungal Keratitis in the Republic of Ireland at 1.53 cases per million population per year. This is significantly higher than the only other population-based estimate, which was 0.32 per million population per year in the United Kingdom in which a degree of failure to report cases is acknowledged. ${ }^{22}$ We believe that our study is less likely to underreport cases as data have been sourced from the seven microbiology laboratories which process corneal specimens directly. Our study accounts only for cases with positive microbiological findings. Cases of suspected fungal keratitis with no positive microbiological findings are not included. About $90.5 \%$ of cases were identified with positive fungal culture, which is the gold standard diagnostic test. This study is limited by the fact that it is retrospective and that a number of cases could be diagnosed in smaller private institutions. We believe that this is unlikely to be the case because significant corneal infections in the Republic of Ireland requiring laboratory analysis are referred to the seven centres in this study. However, as we cannot outrule this, we report our findings as minimum annual incidence. The use of $\mathrm{KOH}$ staining and confocal microscopy in addition to Gram stain, culture, and PCR testing could potentially increase the number of microbiologically confirmed cases of Fungal Keratitis in the future. In contrast to the other studies from temperate climates, we saw an increased incidence in males (57.1\%) compared to females (42.9\%).

While ocular surface disease has generally been reported as the main risk factor and Candida species, the most common organism in cases of fungal keratitis in temperate climates, the recent paper by Ong et al suggests that the microbiological characteristics may be changing. In their study $69.6 \%$ of cases were caused by filamentary fungi and the most common risk factor was contact lens wear, which was present in $57.1 \% .{ }^{20}$ Our study would support that trend. In our study $66.7 \%$ of cases identified filamentary organism and while pre-existing ocular surface disease was the most common risk factor in our study $(18$ cases $(42.9 \%))$, contact lens wear was almost as common $(17$ cases $(40.5 \%))$. We note that 11 of 14 contact lens wearers reported good contact lens hygiene highlighting that fungal keratitis is not a disease that occurs only with poor contact lens hygiene. Seven of eight cases due to trauma were due to a fingernail or inoculation with organic matter as would be expected, but one case surprisingly was due to a metallic foreign body. All cases of fungal keratitis in a pre-existing corneal grafts occurred, while the patient was on topical steroids.

Aspergillus species were the most common organisms isolated overall. This differs from previous studies in temperate climates, all of which identify Candida species as most common with the exception of the recently published series from London, which reported Fusarium to be equally prevalent as Candida and also with the exception of a series from Philadelphia, which identified Fusarium as the most common organism species. The study from Philadelphia incorporated a Fusarium species outbreak associated with the use of ReNu with MoistureLoc contact lens solution. ${ }^{17}$ This contact lens solution-related outbreak was initially reported in Singapore before being identified across the United States. ${ }^{25-29}$ Our study was consistent with previous studies in finding that Candida species were the most common species in patients with chronic ocular surface disease, Fusarium species were the most common in contact lens wearers and Aspergillus species are most common in patients who have suffered corneal trauma.

Visual outcomes have a very broad range from LogMAR - 0.1 (Snellen 6/5) to no perception of light. While this study is not powered to identify statistically significant differences between fungal keratitis caused by different organisms, the two eyes with no identifiable risk factors had an excellent outcome and there is an indication that the eyes in which Fusarium species were 
isolated have better visual outcomes than the eyes with pre-existing ocular surface disease or corneal trauma. There is also an indication that infections due to Fusarium species have a better visual outcome and that infections due to Candida are more likely to result in the necessity for corneal grafts. However this may be due to the underlying risk factors rather than the organism.

Treatment regimens in this study are varied with 12 different topical treatment regimens used for the 42 cases. Voriconazole was used either alone or in combination in 21 of 42 cases $(50.0 \%)$ and amphotericin B was used either alone or in combination in 21 of 42 cases (50.0\%).

Natamycin was used in only 6 of 42 cases (14.3\%). Local injections of antifungal agents into the corneal stroma or anterior chamber were performed in 4 of 42 cases (9.5\%), all of which occurred in one clinical centre, while intravitreal injections of antifungal agents were performed only in a single other separate clinical centre. This shows variation in practices between centres, which is understandable in a relatively rare condition for which there are not widely accepted guidelines.

Eleven of 42 eyes (26.2\%) underwent corneal transplantation in our study. This compares with rates of $12-25 \%$ in Philadelphia, $47.9 \%$ in Iowa, and $30-42 \%$ in London. ${ }^{15-17,19}$ The visual outcomes following corneal grafts varies greatly.

We found in our study that sensitivity testing is not performed routinely by many centres and when it is performed does not appear to follow a consistent protocol. We do see that Candida and Aspergillus species appear to be generally sensitive to all agents tested. A number of Fusarium species show evidence of antifungal resistance including one which was resistant to amphotericin. All samples tested in this study were either fully or partially sensitive to voriconazole. This is similar to the series from London in which sensitivity testing showed voriconazole performed best of the azoles and that all isolates in that study were either fully or partially sensitive to voriconazole. In that study $21 \%$ of filamentary samples were resistant to amphotericin, which compares to $11 \%$ in this study. ${ }^{19}$

In conclusion, this study demonstrates that the minimum incidence of fungal keratitis in the Republic of Ireland is 1.53 cases per million population. Pre-existing ocular surface disease is the most common risk factor followed by contact lens wear and corneal trauma. The most common species is Aspergillus. Voriconazole and amphotericin are the most commonly used topical agents. Visual outcome is very broad ranging from LogMAR -0.10 to no perception of light. The range of visual outcomes remains broad in all subgroups irrespective of risk factors, organism identified, local or systemic treatment, or corneal grafting.

\section{Summary}

What was known before

- A small number of single-institution case series from the United States of America, United Kingdom, and Australia described the epidemiological features of fungal keratitis. Only one nationwide study in the United Kingdom was performed, but this acknowledged significant underreporting of cases.

\section{What this study adds}

- The minimum prevalence of fungal keratitis is nearly five times that reported in the only previous published estimate. This study is more comprehensive than previously published high-quality but limited data as it captures the spectrum of fungal keratitis risk factors, treatment, microbiological findings, and outcomes for an entire nation.

\section{Conflict of interest}

The authors declare no conflict of interest.

\section{Acknowledgements}

We thank Caroline Bailey, Sinead Connolly and Laura Ryan for their assistance in data collection for this study and acknowledge the staff of the microbiology and ophthalmology departments of the Mater Hospital Dublin, The Royal Victoria eye and Ear Hospital Dublin, University Hospital Waterford, Cork University Hospital, Sligo Regional Hospital, University Hospital Galway, University Hospital Limerick and Beaumont Hospital Dublin for their co-operation.

\section{References}

1 Chowdhary A, Singh K. Spectrum of fungal keratitis in North India. Cornea 2005; 24(1): 8-15.

2 Gopinathan U, Garg P, Fernandes M, Sharma S, Athmanathan S, Rao GN. The epidemiological features and laboratory results of fungal keratitis: a 10-year review at a referral eye care center in South India. Cornea 2002; 21(6): 555-559.

3 Srinivasan M, Gonzales CA, George C, Cevallos V, Mascarenhas JM, Asokan B et al. Epidemiology and aetiological diagnosis of corneal ulceration in Madurai, south India. Br J Ophthalmol 1997; 81(11): 965-971.

4 Hagan M, Wright E, Newman M, Dolin P, Johnson G. Causes of suppurative keratitis in Ghana. $\mathrm{Br} J$ Ophthalmol 1995; 79(11): 1024-1028.

5 Xie L, Zhong W, Shi W, Sun S. Spectrum of fungal keratitis in north China. Ophthalmology 2006; 113(11): 1943-1948.

6 Upadhyay MP, Karmacharya PC, Koirala S, Tuladhar NR, Bryan LE, Smolin G et al. Epidemiologic characteristics, predisposing factors, and etiologic diagnosis of corneal ulceration in Nepal. Am J Ophthalmol 1991; 111(1): 92-99.

7 Dunlop AA, Wright ED, Howlader SA, Nazrul I, Husain R, McClellan $\mathrm{K}$ et al. Suppurative corneal ulceration in 
Bangladesh. A study of 142 cases examining the microbiological diagnosis, clinical and epidemiological features of bacterial and fungal keratitis. Aust N Z J Ophthalmol 1994; 22(2): 105-110.

8 Wong TY, Fong KS, Tan DT. Clinical and microbial spectrum of fungal keratitis in Singapore: a 5-year retrospective study. Int Ophthalmol 1997; 21(3): 127-130.

9 Carmichael TR, Wolpert M, Koornhof HJ. Corneal ulceration at an urban African hospital. Br J Ophthalmol 1985; 69(12): 920-926.

10 Laspina F, Samudio M, Cibils D, Ta CN, Fariña N, Sanabria R et al. Epidemiological characteristics of microbiological results on patients with infectious corneal ulcers: a 13-year survey in Paraguay. Graefes Arch Clin Exp Ophthalmol 2004; 242(3): 204-209.

11 Bharathi MJ, Ramakrishnan R, Vasu S, Meenakshi R, Palaniappan R. Epidemiological characteristics and laboratory diagnosis of fungal keratitis. A three-year study. Indian J Ophthalmol 2003; 51(4): 315-321.

12 Maharana P, Sharma N, Nagpal R, Jhanji V, Das S, Vajpayee R. Recent advances in diagnosis and management of mycotic keratitis. Indian J Ophthalmol 2016; 64(5): 346-357.

13 Verma S, Sharma V, Kanga A, Sharma R, Angrup A, Mokta K et al. Current spectrum of oculomycosis in North India: a 5-year retrospective evaluation of clinical and microbiological profile. Indian J Med Microbiol 2016; 34(1): 72-75.

14 Ritterband DC, Seedor JA, Shah MK, Koplin RS, McCormick SA. Fungal keratitis at the New York eye and ear infirmary. Cornea 2006; 25(3): 264-267.

15 Rogers GM, Goins KM, Sutphin JE, Kitzmann AS, Wagoner MD. Outcomes of treatment of fungal keratitis at the University of Iowa Hospitals and Clinics: a 10-year retrospective analysis. Cornea 2013; 32(8): 1131-1136.

16 Tanure MA, Cohen EJ, Sudesh S, Rapuano CJ, Laibson PR. Spectrum of fungal keratitis at Wills Eye Hospital, Philadelphia, Pennsylvania. Cornea 2000; 19(3): 307-312.

17 Yildiz EH, Abdalla YF, Elsahn AF, Rapuano CJ, Hammersmith KM, Laibson PR et al. Update on fungal keratitis from 1999 to 2008. Cornea 2010; 29(12): 1406-1411.
18 Bhartiya P, Daniell M, Constantinou M, Islam FM, Taylor HR. Fungal keratitis in Melbourne. Clin Exp Ophthalmol 2007; 35(2): 124-130.

19 Galarreta DJ, Tuft SJ, Ramsay A, Dart JK. Fungal keratitis in London: microbiological and clinical evaluation. Cornea 2007; 26(9): 1082-1086.

20 Ong HS, Fung SS, Macleod D, Dart JK, Tuft SJ, Burton MJ. Altered patterns of fungal keratitis at a London Ophthalmic Referral Hospital: an Eight-Year Retrospective Observational Study. Am J Ophthalmol 2016; 168: 227-236.

21 Rosa Jr RH, Miller D, Alfonso EC. The changing spectrum of fungal keratitis in south Florida. Ophthalmology 1994; 101(6): 1005-1013.

22 Tuft SJ, Tullo AB. Fungal keratitis in the United Kingdom 2003-2005. Eye (Lond) 2009; 23(6): 1308-1313.

23 Holladay JT. Visual acuity measurements. J Cataract Refract Surg 2004; 30(2): 287-290.

24 Central Statistics Office GoI. This is Ireland, Highlights from Census 2011, Part 1. 2012. Available at: http:/ /www.cso.ie/ en/census/census2011reports/census2011thisisirelandpart1/.

25 Khor WB, Aung T, Saw SM, Wong TY, Tambyah PA, Tan AL et al. An outbreak of Fusarium keratitis associated with contact lens wear in Singapore. JAMA 2006; 295(24): 2867-2873.

26 Alfonso EC, Cantu-Dibildox J, Munir WM, Miller D, O'Brien TP, Karp CL et al. Insurgence of Fusarium keratitis associated with contact lens wear. Arch Ophthalmol 2006; 124(7): 941-947.

27 Gorscak JJ, Ayres BD, Bhagat N, Hammersmith KM, Rapuano CJ, Cohen EJ et al. An outbreak of Fusarium keratitis associated with contact lens use in the northeastern United States. Cornea 2007; 26(10): 1187-1194.

28 Bernal MD, Acharya NR, Lietman TM, Strauss EC, McLeod SD, Hwang DG. Outbreak of Fusarium keratitis in soft contact lens wearers in San Francisco. Arch Ophthalmol 2006; 124(7): 1051-1053.

29 Jeng BH, Hall GS, Schoenfield L, Meisler DM. The fusarium keratitis outbreak: not done yet? Arch Ophthalmol 2007; 125(7): 981-983. 